\title{
EVALUACIÓN TOXICOLÓGICA DEL AGUA Y LOS SEDIMENTOS EN EL EMBALSE LA FE, COLOMBIA
}

\section{TOXICITY ASSESSMENT OF WATER AND SEDIMENT SAMPLES \\ IN LA FE RESERVOIR, COLOMBIA}

\author{
${ }^{1 *}$ Amaringo F., ${ }^{1}$ Molina $F$. \\ ${ }^{1}$ Grupo de Investigación en Gestión y Modelación Ambiental GAIA, Escuela Ambiental, \\ Facultad de Ingeniería, Universidad de Antioquia UdeA, Calle 70 No. 52-21, Medellín, Colombia.
}

${ }^{1}$ fredy.amaringo@udea.edu.co; ${ }^{1}$ francisco.molina@udea.edu.co

*Autor de correspondencia

\section{RESUMEN}

Contextualización del tema: la calidad del agua de los embalses destinados al tratamiento y suministro de agua potable se debe monitorear mediante análisis de toxicidad del agua y sedimentos, ya que los sedimentos pueden actuar como fuente de contaminantes orgánicos, de tal forma que durante procesos de mezcla y transporte estos contaminantes pueden quedar disponibles en los ecosistemas acuáticos.

Vacío de investigación: debido a que estos contaminantes esta en el orden de trazas, se hace necesaria la utilización de metodos de muestreo pasivo que permitan la acumulación de compuestos hidrofóbicos en dispositivos de membranas semipermeables (SPMDs) para la posterior evaluación.

Propósito del estudio: en este estudio, se evaluó la toxicidad de extractos dializados de muestreo pasivo tipo SPMD y de sedimentos, mediante ensayos Microtox ${ }^{\circledR}$, usando la bacteria Vibrio fischeri.

Metodología: los 24 extractos analizados se obtuvieron a partir de dispositivos de membranas semipermeables (SPMDs) previamente desplegadas durante 21 días en seis estaciones en el cuerpo de agua del embalse La Fe, Antioquia, durante cuatro campañas de muestreo. Los extractos se sometieron a ensayo mediante el Microtox Basic Test. La evaluación del potencial de toxicidad del sedimento del embalse se analizó de igual forma en 12 muestras, mediante ensayos de toxicidad aguda con Microtox $®$, aplicando el protocolo Solid Phase Test (SPT). Asimismo, se determinaron los parametros fisicoquímicos del agua $\mathrm{pH}$, temperatura, conductividad eléctrica, oxígeno disuelto y turbidez.

Resultados: los resultados de los ensayos mostraron que los extractos de SPMD 
recolectados en el cuerpo de agua no presentaron toxicidad, mientras que los elutriados de sedimentos presentaron una toxicidad moderada o baja.

Palabras clave: contaminantes; calidad del agua; sedimentos; toxicidad aguda.

\section{ABSTRACT}

Contextualization: To guarantee the quality of the water in the reservoirs destined to the treatment and supply of drinking water, toxicity analyzes of the water and sediments must be carried out periodically, due to its can be reservoirs of the organic pollutants that during remobilization processes could make those available in the aquatic ecosystems.

Knowledge gap: Because these contaminants concentrations are found in traces (ng/L), it is necessary to use passive sampling methods that allow the accumulation of hydrophobic compounds in semi-permeable membrane devices (SPMDs) for subsequent evaluation.

Purpose: The purpose of this study was to evaluate the toxicity of samples from SPMD-type passive sampling extracts and sediments by the use Microtox ${ }^{\circledR}$ assays using the Vibrio fischeri bacteria.

Methodology: The 24 extracts used in the trial were obtained from semi-permeable membrane devices (SPMDs) previously deployed for 21 days in 6 stations located in the reservoir of the La Fe, Antioquia, in four sampling campaigns. The extracts were tested by Microtox Basic Test medium. The evaluation of the toxicity potential of the reservoir sediment was also analyzed by means of acute toxicity tests with Microtox $®$ applying the Solid Phase Test (SPT) protocol as well as the physicochemical parameters of water such as $\mathrm{pH}$, temperature, electrical conductivity, dissolved oxygen and turbidity.

Results and conclusions: The results of the toxicity tests indicate that the analysis of the extracts of SPMD collected in water reservoir La Fe does not present toxicity, while those fed by sediments presented moderate or low toxicity.

Keywords: water quality; acute toxicity; sediments. 


\section{RESUMEN GRÁFICO}
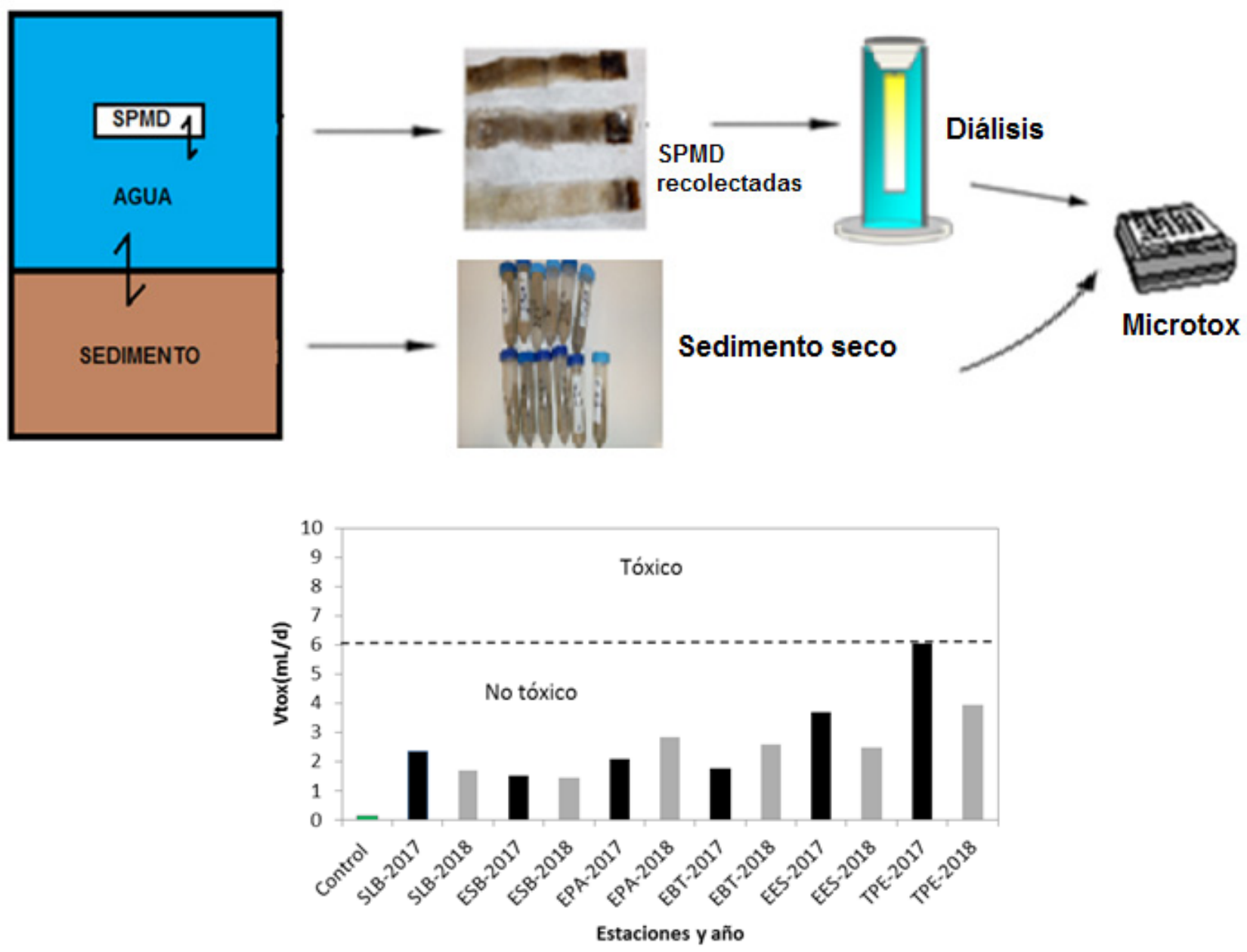

Fuente: autores

\section{INTRODUCCIÓN}

La evaluación de la toxicidad representa una herramienta fundamental en el control y manejo ambiental de los vertimientos de contaminantes en los ecosistemas acuáticos (Beg \& Ali, 2008). Debido a que los compuestos orgánicos son contaminantes con concentraciones en el orden de trazas ( $\mathrm{ng} / \mathrm{L}$ ), se hace necesaria la utilización de métodos que permitan la acumulación de compuestos hidrofóbicos en soportes para su posterior evaluación.

Uno de los métodos en la toma de muestras para la evaluación de toxicidad de contaminantes en cuerpos de agua son los dispositivos de muestreo pasivo (PSD) (Allan et al., 2010) El principio de la técnica de muestreo pasivo consiste en el despliegue de un dispositivo de membrana semipermeable (SPMD) en el cuerpo de agua, durante un período definido de tiempo. En dicho periodo, los contaminantes se difunden libremente a través de la membrana y se acumulan por medio de procesos difusivos y de adsorción (Charriau et al., 2016).

Las membranas semipermeables (SPMD), son dispositivos que permiten un muestreo pasivo in situ, las cuales contienen en su interior Trioleína, cuyo carácter lipofílico, permite el 
análisis de contaminantes orgánicos persistentes (POPs) u otros contaminantes orgánicos hidrofóbicos con el coeficiente de partición octanol/agua mayor a 3 (log Kow $\geq 3$ ) (Huckins et al., 2006; Narvaez \& Molina, 2012). Por ejemplo, entre los compuestos analizados, se encuentran los hidrocarburos aromáticos policíclicos (PAH) bifenilos policlorados (PCB), pesticidas organoclorados y contaminantes orgánicos persistentes (POP) (Forsberg et al., 2014); (Karacık et al., 2013) ; (Narvaez, Lopez, \& Molina, 2013); (Lohmann et al., 2012).

En este sentido, Johnson et al.,(2000) desarrollaron una metodología que permite integrar el sistema de ensayos de toxicidad aguda Microtox $^{\circledR}$ con extractos dializados de dispositivos de membrana semipermeable (SPMD-TOX) (Johnson et al., 2000). Esta metodología, se fundamenta en la bioluminiscencia natural de la bacteria marina Photobacterium phosphoreum, también conocida como Vibrio fischeri que, en presencia de agentes contaminantes, reduce la bioluminiscencia natural, y por lo tanto, la toxicidad se expresa como concentración efectiva, que reduce más del $50 \%$ de la luminiscencia inicial $\left(\mathrm{EC}_{50}\right.$ )(Fernández-piñas et al., 2014);(Onorati \& Mecozzi, 2004). El análisis de toxicidad por medio de Microtox ${ }^{\circledR}$, ha sido aplicado por más de 40 años, ya que es un método rápido, simple y sensible para la evaluación del riesgo por exposición a contaminantes transmitidos a través del agua antibióticos como la amoxicilina y ciprofloxacina; antibacteriales; contaminantes atmosféricos; pesticidas; herbicidas; plantas medicinales; metales pesados como zinc, cobre, plomo, plata y mercurio, cadmio (II) y cromo (VI) (Palma et al., 2010);(Ji et al., 2013);(Grinten, Pikkemaat, \& Brandhof, 2010);(Kováts \& Horváth, 2016);(Mansour, Abdel-hamid, \& Ibrahim,
2015);(Palma et al., 2008);(Gatidou, Stasinakis, \& Iatrou, 2014);(Kovats et al., 2011);(Gonçalves et al., 2015);(Fulladosa et al., 2005);(Fulladosa, Murat, \& Villaescusa, 2005).

En este estudio, se utilizaron Microtox $®$ y extractos dializados de membranas SPMD desplegadas para conocer las condiciones ambientales de un embalse que suministra agua a la planta de potabilización La Ayurá, que abastece la zona sur del Valle de Aburrá, Colombia.

\section{MATERIALES Y MÉTODOS}

\section{1. Área de estudio}

El embalse La Fe tiene un área de 1,73 Km²; se ubica en el municipio de El Retiro en el departamento de Antioquia; Tiene una capacidad de 11.58 millones de $\mathrm{m}^{3} \mathrm{y}$ el área de sus tributarios abarca $78 \mathrm{Km}^{2}$; recibe aguas de las quebradas Las Palmas, San Luis-Boquerón, Espíritu Santo y aportes de los ríos PantaniIlo, Piedras y Buey por mecanismo de bombeo (Salazar, 2017). Se encuentra localizado en una zona de alta montaña, de topografía encañonada y es el resultado de dos fases de construcción; la primera, finalizada en 1967; y la segunda debido a una ampliación en 1974 (Florez et al., 2017). Debido a la influencia y meteorización de las rocas del batolito antioqueño los suelos son arcillosos, de color rojo y pardo rojizo, ricos en materia orgánica y nitrógeno total, muy ácidos ( $\mathrm{pH}$ entre 4.4 y 4.5) y grandes fijadores de fosforo (Arias \& Ramirez, 2009). Debido a las actividades agrícolas de la región y a la densificación inmobiliaria, los residuos domésticos y agroindustriales, ingresan de manera directa o indirecta a las quebradas y ríos llegando finalmente al embalse. El embalse La Fe es un importante reservorio de agua para el Valle de Aburrá y abastece agua potable para más de un millón de habitantes. 


\subsubsection{Trabajo de campo}

Se ubicaron las estaciones de monitoreo en las principales cuencas tributarias aportantes al embalse y algunas fueron ubicadas en zonas de transición entre los tributarios y el sistema léntico del embalse (Figura 1, Tabla 1).

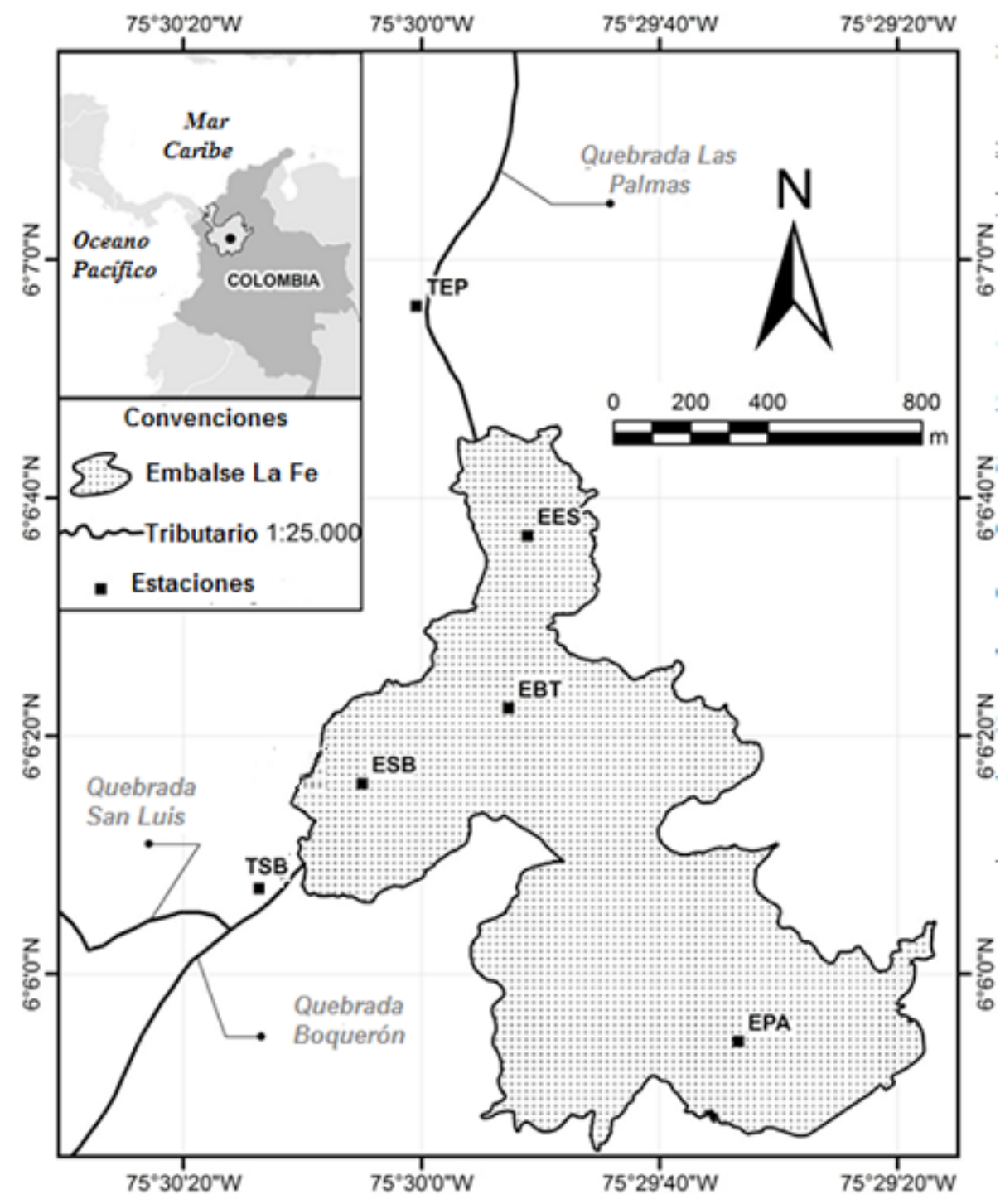

Figura 1. Mapa de las estaciones de muestreo en el embalse La Fe, Colombia. Tributario San Luis-Boquerón (TSB), Tributario Palmas-Espíritu Santo (TPE), zona de entrada de las quebradas Palmas y Espíritu Santo al embalse (EES), Torre de captación (EBT), zona de entrada de las quebradas San Luis y Boquerón al embalse (ESB) y zona de entrada al embalse del bombeo del rio Pantanillo (EPA).

Fuente: Autores 
Tabla 1. Coordenadas de ubicación de las estaciones de muestreo.

\begin{tabular}{c|c|c}
\hline \multicolumn{1}{c|}{$\begin{array}{c}\text { Estación } \\
\text { TSB }\end{array}$} & Longitud & Latitud \\
\hline TEP & $\mathrm{N} 6^{\circ} 6^{\prime} 16.786^{\prime \prime} 56.107^{\prime \prime}$ & $075^{\circ} 30^{\prime} 13.168^{\prime \prime}$ \\
\hline EES & $N 6^{\circ} 6^{\prime} 36.813^{\prime \prime}$ & $0.425^{\prime \prime}$ \\
\hline EBT & $N 6^{\circ} 6^{\prime} 22.342^{\prime \prime}$ & $075^{\circ} 29^{\prime} 51.075^{\prime \prime}$ \\
\hline ESB & $N 6^{\circ} 6^{\prime} 16.013^{\prime \prime}$ & $075^{\circ} 29^{\prime} 52.697^{\prime \prime}$ \\
\hline EPA & $N 6^{\circ} 5^{\prime} 54.353^{\prime \prime}$ & $075^{\circ} 30^{\prime} 4.98^{\prime \prime}$ \\
\hline
\end{tabular}

Fuente: Autores

El muestreo se realizó mediante el despliegue de tres membranas tipo SPMD, protegidas de condiciones externas por una canastilla de acero inoxidable, que permite adicionalmente anclar el dispositivo. Estas canastillas se instalaron para las estaciones de tributarios en tuberías perforadas adosadas a estructuras hidráulicas existentes (pilas de puentes o muros laterales), y en las estaciones de embalse se fijaron en boyas que evitan su pérdida, lo que asegura una profundidad adecuada $(2 \mathrm{~m})$ para garantizar un contacto continuo entre la membrana y el agua durante el tiempo estipulado de muestreo (Narvaez \& Molina, 2012). Para este estudio de caso, el tiempo de exposición de las membranas en el cuerpo de agua fue de 21 días durante octubre y noviembre de 2017 y 2018. Además, se muestrearon los sedimentos superficiales $(<5 \mathrm{~cm}$ ) utilizando una draga Ekman en las seis estaciones el día de despliegue de las membranas SPMD. Los parámetros fisicoquímicos $\mathrm{pH}$, conductividad, temperatura, oxígeno disuelto y turbidez se midieron en un equipo multiparámetrico HANNA 9829.

\subsection{Reactivos y estándares}

En la extracción y análisis se usaron solventes tales como acetona, hexano y DMSO (Pureza > 99\%), marca Sigma-Aldrich. Por otro lado, la solución de reconstitución, cloruro de sodio al $2 \%$, el ajuste osmótico al $22 \%$ y el cultivo de la bacteria bioluminiscente Vibrio fischeri se usaron en los ensayos de toxicidad marca Azur Environmental, (Carlsbad, CA). Se usaron las membranas SPMD marca Environmental Sampling Technologies EST-Lab (St. Joseph, MO). Las membranas de polietileno de baja densidad tienen un área superficial aproximada de $450 \mathrm{~cm}^{2}$ y un contenido de $1 \mathrm{~mL}$ trioleína (99\% de pureza).

\subsection{Ensayo de toxicidad utilizando SPMD-TOX.}

Desde hace tres décadas, los muestreadores pasivos tipo SPMD se han venido utilizando en el monitoreo de contaminantes orgánicos debido a la posibilidad de integración analítica con las evaluaciones del potencial tóxico de los extractos obtenidos, lo cual no es posible con los métodos de monitoreo puntual (Petty et al., 2000). Para el ensayo de toxicidad SPMD-TOX, se aplicó el protocolo descrito por (Johnson et al., 2000) en el cual las membranas recolectadas se lavaron con un cepillo para retirar la biopelícula y los residuos. Las membranas se almacenaron en papel aluminio y se transportaron al laboratorio del grupo de Investigación GAIA de la Universidad de Antioquia en bolsas selladas, para evitar contaminación cruzada. En el laboratorio, se lavaron las membranas con una solución de $\mathrm{HCl}$ al $10 \%$ para remover las sales inorgánicas y el biofilm adherido en la superficie, seguido de un lavado con agua desionizada e isopropanol para remover los 
residuos de agua. Finalmente, las membranas fueron dializadas en hexano durante 24 horas y el extracto hexánico se concentró con nitrógeno hasta obtener $1 \mathrm{~mL}$. La solución obtenida se pasó a través de una columna de fase sólida, la cual fue eluída con acetona y se concentró nuevamente el extracto con dimetilsulfosido (DMSO) (Figura 2). En el extracto reconstituido en DMSO, se procedió midiendo el $\mathrm{pH}$ de cada muestra, para establecer el rango óptimo para la medición de los ensayos de Microtox ${ }^{\circledR}$ de soluciones acuosas entre 6.0 - 8.5 (Tabla 2).

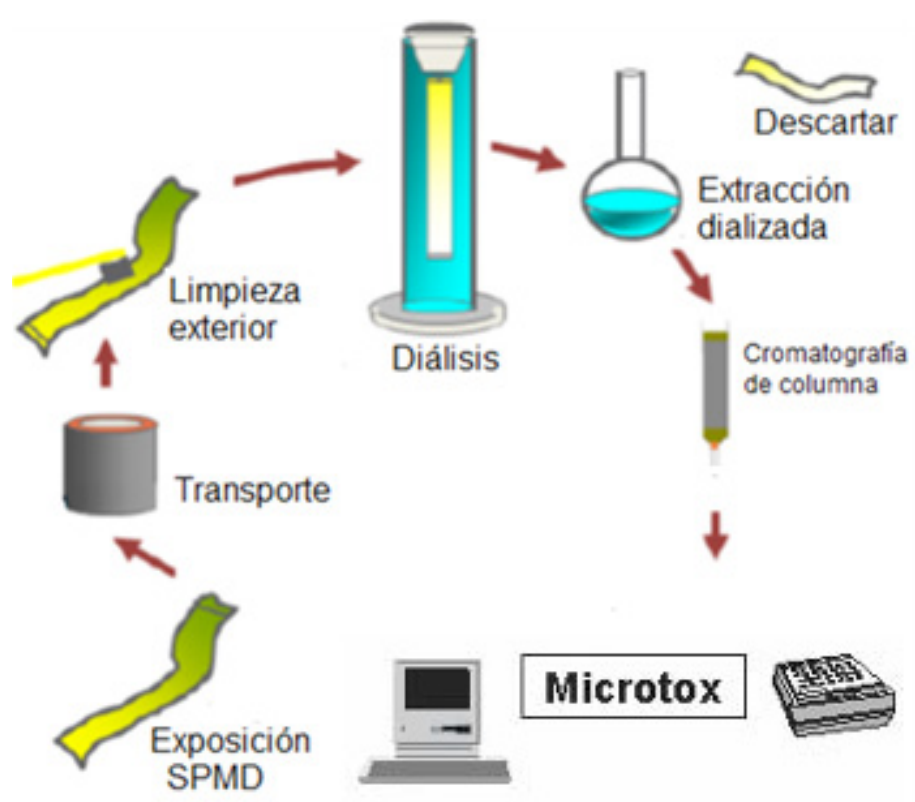

Figura 2. Protocolo evaluación de toxicidad utilizando SPMD-TOX.

Fuente: (Modificado de Johnson., 2005).

Las muestras se colocaron en cubetas con las bacterias Vibrio fischeri en una solución de reconstitución de $1 \mathrm{~mL}$. Posteriormente, se añadieron $500 \mu \mathrm{L}$ del diluyente en cada cubeta con $250 \mu \mathrm{L}$ de ajuste osmótico y luego se transfirieron las muestras a una serie de cubetas preparando cuatro diluciones en serie 1:2 del sobrenadante. Se realizaron réplicas de los controles y las cuatro diluciones. Las muestras y las réplicas se prepararon en las cubetas, luego se transfirieron las bacterias reconstituidas a cada celda y se realizó la medición de la intensidad de luminiscencia con el equipo Microbic Model 500 a tiempos de 0, 5, 15 y 30 minutos, hallando la concentración efectiva al
$50 \%($ EC50) y el porcentaje de efecto en la bacteria. Todas las muestras, fueron analizadas por duplicado, usando 4 concentraciones y un control negativo.

Teniendo en cuenta que se utilizan solventes orgánicos en la extracción de los contaminantes, se dificulta la evaluación de la toxicidad de los extractos dializados obtenidos de los SPMD (Koci, Mlejnek, \& Kochankova, 2003). Se aplicó $V_{\text {tox }}$ para remediar estas interferencias de los solventes de extracción. $V_{\text {tox }}$ representa el volumen promedio teórico que se necesita para la dilución de los contaminantes y para que se absorban en una 
membrana durante el promedio de días de exposición, y así obtener una solución con una concentración efectiva $\mathrm{EC}_{50}$ para evaluar la toxicidad (Huckins et al., 2000).

Para esto, se define la ecuación (1):

$$
V_{\text {tox }}(50)=\frac{1}{m * E C 50 * d}=(1)
$$

Donde, $\mathrm{m}$ es la concentración de membranas extraídas en la mezcla de solventes, expresado como número de membranas semipermeables en $\mathrm{mL}$, en nuestro caso $\mathrm{m}=3 / \mathrm{mL}$ en cada estación; d es el número de días de exposición de las membranas en el cuerpo de agua, en nuestro caso 21 días; EC50 es el $50 \%$ de la concentración efectiva del extracto en el organismo a evaluar ( $\mathrm{mL} / \mathrm{L})$. Debido a esto a todos los valores $V_{\text {tox }}$ se asignan el porcentaje de concentración efectivo entre paréntesis utilizado. Al resolver la ecuación 1 , se obtienen los resultados en $\mathrm{L} / \mathrm{d}$, para cuestiones prácticas lo convertimos en $\mathrm{mL} / \mathrm{d}$ utilizando factores de conversión. Un valor alto $r$ de $V_{\text {tox }}$ es un indicativo de contaminación ambiental (Koci et al., 2003).

\subsection{Ensayo de toxicidad de sedimentos en Fase sólida (SPT) con Microtox ${ }^{\circledR}$.}

Se secaron los sedimentos recolectados en cada estación a temperatura ambiente (aproximadamente a $25^{\circ} \mathrm{C}$ ) para evitar la evaporación de los compuestos orgánicos semivolatiles (SVOC), tamizados en una malla ASTM \# 200, para obtener un tamaño de partícula inferior a $75 \mu \mathrm{m}$ y luego se almacenaron en bolsas de plástico y se guardaron a una temperatura de $-20^{\circ} \mathrm{C}$. Posteriormente, se pesó una muestra de $400 \mathrm{mg}$ de sedimento seco y se disolvieron en $1 \mathrm{~mL}$ de diluyente, preparando una solución de la bacteria con la solución de reconstitución, y el ajuste osmótico se agitó en un agitador mecánico durante cinco minutos. La muestra se transfirió a una serie de tubos de fase sólida, y se prepararon nueve diluciones en serie 1:2 del sobrenadante, incluidos los controles. Se realizaron dos réplicas de los controles y las 9 diluciones, las muestras y las réplicas se prepararon en los tubos de fase sólida, luego se transfirieron las bacterias reconstituidas a cada tubo de fase sólida y se incubaron las bacterias en el tubo durante 20 minutos. Finalmente, se insertaron en los tubos de fase sólida las columnas de filtros y junto con la solución fueron filtradas las bacterias. Luego, se transfirieron $500 \mu \mathrm{L}$ de la solución a las cubetas de medición del analizador Microtox ${ }^{\circledR} 500$ y se obtuvieron lecturas de luminiscencia para generar valores de concentración efectiva EC50 a tiempos de 5,15 y 30 minutos. De igual forma, todas las muestras fueron analizadas por duplicado.

\subsection{Análisis de los datos}

Para garantizar la calidad de los datos, se procesaron los resultados en el software estadístico Modern Water Microtox Omni ${ }^{\circledR}$, versión 4.3, para generar la concentración efectiva EC50 y el porcentaje de efecto, obteniendo los intervalos de confianza (CI) y coeficiente de correlación $\left(R^{2}\right)$ de las muestras. Los valores obtenidos, se consideraron como válidos cuando el CI no excede el $30 \%$ y $\mathrm{R}^{2}$ se aproxima a uno (Grigson, Cheong, \& Way, 2006).

\section{RESULTADOS Y DISCUSIÓN}

\subsection{Parámetros fisicoquímicos}

$\mathrm{El} \mathrm{pH}$ de todas las estaciones tuvo valores entre 6,21 y 8,05 , lo cual es característico de cuerpos de agua que se utilizan para el tratamiento y suministro de agua potable, ya que este parámetro es importante en la estabilidad de los compuestos orgánicos (WHO, 1997).

La conductividad eléctrica (CE) mide la concentración de los sólidos disueltos totales y los iones principales. Normalmente, la CE de los cuerpos de agua dulce está en el rango de 10$1000 \mu \mathrm{S} / \mathrm{cm}$ (APHA, 2017). En este estudio, los 
valores de CE estuvieron en el rango de $26 \mu \mathrm{S} /$ $\mathrm{cm}$ a $87 \mu \mathrm{S} / \mathrm{cm}$, resaltando que el valor mínimo se encuentra en el tributario SLB donde no se evidencia ninguna actividad antrópica y el valor máximo en el tributario TPE, donde hay presencia de talleres de alfarería, unidades residenciales y vías intermunicipales de alto tráfico vehicular, que posiblemente incrementan la presencia de solidos disueltos e iones. En las demás estaciones dentro del embalse, los valores de conductividad variaron entre 42 $\mu \mathrm{S} / \mathrm{cm}$ y $61 \mu \mathrm{S} / \mathrm{cm}$.

Tabla 2. Parámetros fisicoquímicos del agua en las estaciones de muestreo del Embalse La Fe.

\begin{tabular}{|c|c|c|c|c|c|c|c|c|}
\hline Estación & TSB & TPE & EES & EBT & ESB & EPA & Media & D.E \\
\hline pH & 6,21 & 6,38 & 6,68 & 6,77 & 6,61 & 8,05 & 6,78 & 0,653 \\
\hline $\mathbf{T}\left({ }^{\circ} \mathbf{C}\right)$ & 16,9 & 16,5 & 21,8 & 18,6 & 21,5 & 21,4 & 19,5 & 2,42 \\
\hline Conductividad $(\mu \mathrm{S} / \mathrm{cm})$ & 26 & 87 & 48 & 61 & 42 & 45 & 51,5 & 20,7 \\
\hline OD (mg/L) & 3,86 & 5,75 & 5,02 & 5,49 & 5,27 & 7,52 & 5,485 & 1,19 \\
\hline Turbidez (UTN) & 13 & 44 & 23 & 23 & 21 & 23 & 24,5 & 10,3 \\
\hline
\end{tabular}

Tributario San Luis-Boquerón (TSB), Tributario Palmas-Espíritu Santo (TPE), zona de entrada de las quebradas Palmas y Espíritu Santo al embalse (EES), Torre de captación (EBT), zona de entrada de las quebradas San Luis y Boquerón al embalse (ESB) y zona de entrada al embalse del bombeo del rio Pantanillo (EPA).D.E: Desviación estándar.

Fuente: Autores

\subsection{Evaluación de la toxicidad con SPMD} El protocolo de muestras SPMD-TOX que se desplegaron en el embalse demostró que los valores de $V_{\text {tox }}(50)$ superiores a $6,35 \mathrm{~mL} / \mathrm{d}$ se consideran tóxicos (Johnson, 2005). Ningún valor de las muestras evaluadas en esta investigación sobrepasa este límite; sin embargo, los valores más cercanos correspondieron a la estación TPE en el año 2017 y 2018 , es decir 6,05 mL/d y 3,94 $\mathrm{mL} / \mathrm{d}$ respectivamente, indican la relación directa entre las actividades antropogénicas y la toxicidad de las muestras tomadas en esta estación (Tabla 3). Se define el limite admisible de $6,35 \mathrm{~mL} / \mathrm{d}$, debido a que $\mathrm{n}=3$ y $\mathrm{d}=21$ y reemplazando en la ecuación 1 , en donde valores superiores a $2.5 \mathrm{mgSPMD} /$ $\mathrm{mL}$ son considerados como tóxicos (Figura 3) (Johnson, 2005). 
Tabla 3. Valores de $\mathrm{pH}, \mathrm{EC}_{50}$ y $V_{\text {tox }}$ en todas las estaciones donde se desplegaron las SPMD.

\begin{tabular}{|c|c|c|c|c}
\hline Estación-año & PH & EC $_{\mathbf{5 0}}$ & $\boldsymbol{V}_{\text {tox }}$ & $\mathbf{R}^{\mathbf{2}}$ \\
\hline TSB-2017 & 7,48 & 6,71 & 2,36 & 0,9869 \\
\hline TSB-2018 & 7,28 & 9,34 & 1,69 & 0,9732 \\
\hline ESB-2017 & 7,74 & 10,45 & 1,51 & 0,9453 \\
\hline ESB-2018 & 7,83 & 11,12 & 1,42 & 0,9479 \\
\hline EPA-2017 & 6,99 & 7,59 & 2,09 & 0,9840 \\
\hline EPA-2018 & 7,11 & 5,57 & 2,85 & 0,9313 \\
\hline EBT-2017 & 7,18 & 9,06 & 1,75 & 0,9807 \\
\hline EBT-2018 & 7,40 & 6,16 & 2,57 & 0,9961 \\
\hline EES-2017 & 7,15 & 4,31 & 3,68 & 0,9975 \\
\hline EES-2018 & 7,46 & 6,4 & 2,48 & 0,9864 \\
\hline TPE-2017 & 6,88 & 2,62 & 6,05 & 0,9806 \\
\hline TPE-2018 & 7,08 & 4,02 & 3,94 & 0,9962 \\
\hline Media & 7,30 & 6,94 & 2,70 & 1,32 \\
\hline Desviación estándar & 0,292 & 2,65 & -- \\
\hline
\end{tabular}

Fuente: Autores

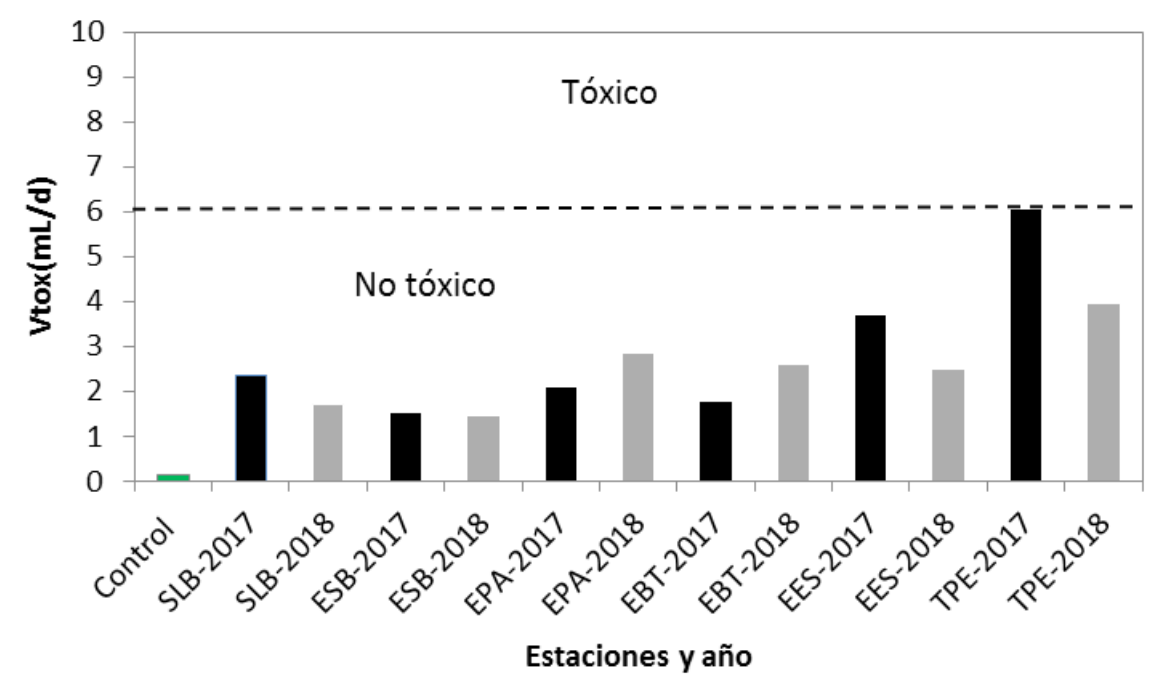

Figura 3. Toxicidad de extractos SPMD en las estaciones de muestreo.

Fuente: Autores

Diversas investigaciones han utilizado los extractos de SPMD para la evaluación de toxicidad aguda con diferentes sistemas como el Microtox ${ }^{\circledR}$, obteniendo resultados representativos de toxicidad que permiten un monitoreo y gestión medioambiental en los sitios de estudio (Sabaliunas et al., 2000)Mutatox(TM. Además, entidades gubernamentales como la United States Geological Survey (USGS) han encontrado en las membranas SPMD una herramienta esencial para el monitoreo de los contaminantes en ríos y lagos, reportando toxicidad elevada en arroyos y ríos de la ciudad de Milwaukee en el año 2007(USGS, 2014).

\subsection{Ensayos de toxicidad en sedimentos}

Considerando que diferentes compuestos orgánicos persistentes (Abbas et al., 2018) pueden 
presentar un efecto inhibitorio a la bacteria $\mathrm{Vi-}$ brio fischeri, se estableció una clasificación de toxicidad en sedimentos que se expresa en $\mathrm{EC}_{50}$, o porcentaje de efecto (Kwan \& Dutka, 1996). Se denominan como tóxicos cuando, en el bioensayo, el $\mathrm{EC}_{50}$ es menor que $5.000 \mathrm{mg} / \mathrm{L}$ $\left(\mathrm{EC}_{50}<0,5 \%\right)$, moderadamente tóxicos entre $5.000 \mathrm{mg} / \mathrm{L}$ y $10.000 \mathrm{mg} / \mathrm{L}\left(\mathrm{EC}_{50}>0,5 \leq 1 \%\right)$ y no tóxicos mayores que $10.000 \mathrm{mg} / \mathrm{L}\left(\mathrm{EC}_{50}>1,0 \%\right)$ (Beg \& Ali, 2008). Al evaluar la toxicidad mediante el sistema Microtox $®$ usando el protocolo Solid Phase Test (SPT). Los valores de $\mathrm{EC}_{50}$ mayor que $100.000 \mathrm{mg} / \mathrm{L}$ se consideran no tóxicos para la bacteria Vibrio fischeri, de tal forma que en las muestras de sedimentos del embalse La Fe en las estaciones correspondientes a los tributarios TSB y TPE tuvieron valores superiores a 10.000 $\mathrm{mg} / \mathrm{L}$ y calificaron como no tóxicos durante el periodo de estudio (Tabla 3). Para las estaciones en el interior del embalse, los sedimentos resultaron moderadamente tóxicos, con valores $\mathrm{EC}_{50}$ de $5.246 \mathrm{mg} / \mathrm{L}$ para EPA y $9.211 \mathrm{mg} / \mathrm{L}$ para EBT.

Hay que destacar que en la estación EPA en el 2017 resultó la única estación considerada como tóxica (4.734 mg/L) y, en el año 2018, como moderadamente tóxica $(5.246 \mathrm{mg} / \mathrm{L})$. Lo anterior implica una carga contaminante alta en el sedimento proveniente del bombeo del rio Pantanillo (Figura 4). Los resultados en las estaciones no mostraron diferencias significativas entre 2017 y 2018 con excepción de la estación TPE, la cual tuvo mayor toxicidad en 2017. Las demás estaciones estuvieron en el rango de $95 \%$ de confianza, y ningún valor de confianza sobrepasó el 30 \% lo que garantiza la calidad de los datos (Tabla 4).

Tabla 4. Valores de $\mathrm{EC}_{50}$ y $\mathrm{CI}$ en todas las estaciones donde se recolectaron los sedimentos.

\begin{tabular}{|c|c|c|c|c|}
\hline Estación/año & $\mathrm{EC}_{50}(\mathrm{mg} / \mathrm{L})$ & CI ( $95 \%$ confianza) & $\mathbf{R}^{2}$ & Clasificación \\
\hline Control & $10 \%$ & ---- & --- & No Tóxico \\
\hline Referencia & $1734(0,17 \%)$ & $(1576-1967)$ & 0,9862 & Tóxico \\
\hline TSB-OCT/17 & $68396(6,83 \%)$ & $(57458-81414)$ & 0,9836 & No tóxico \\
\hline TSB-OCT/18 & $50239(5,02 \%)$ & $(47428-53217)$ & 0,9954 & No tóxico \\
\hline ESB-OCT/17 & $7562(0,76 \%)$ & $(7001-8168)$ & 0,9929 & Moderadamente tóxico \\
\hline ESB-OCT/18 & $7669(0,77 \%)$ & $(6588-8928)$ & 0,9724 & Moderadamente tóxico \\
\hline EPA-OCT/17 & $4734(0,47 \%)$ & $(4112-5450)$ & 0,9761 & Tóxico \\
\hline EPA-OCT/18 & $5246(0,52 \%)$ & $(4727-5821)$ & 0,9881 & Moderadamente tóxico \\
\hline EBT-OCT/17 & $16166(1,62 \%)$ & $(13743-19017)$ & 0,9723 & No tóxico \\
\hline EBT-OCT/18 & $9211(0,92 \%)$ & $(7690-11104)$ & 0,9574 & Moderadamente tóxico \\
\hline EES-OCT/17 & $26865(2,69 \%)$ & $(25806-27966)$ & 0,9975 & No tóxico \\
\hline EES-OCT/18 & $30398(3,04 \%)$ & $(27061-34146)$ & 0,9864 & No tóxico \\
\hline TPE-OCT/17 & $13254(1,33 \%)$ & $(11667-15058)$ & 0,9806 & No tóxico \\
\hline TPE-OCT/18 & $58155(5,82 \%)$ & $(54941-61558)$ & 0,9962 & No tóxico \\
\hline Media & $24824(2,48 \%)$ & & & \\
\hline D.E & $22406(2,24 \%)$ & & & \\
\hline
\end{tabular}

D.E: Desviación estándar. Fuente: autores 
Diversas investigaciones han evaluado ensayos de toxicidad (Jarque et al., 2016) y cinética de contaminantes en sedimentos (Blaha et al., 2009) mediante el método Microtox $\AA$, obteniendo resultados significativos que permiten un monitoreo y gestión medioambiental en los sitios de estudio (Burton et al., 2001);(Svenson et al., 1996). Con el uso de este método se pudo determinar que los sedimentos en el curso alto del rio Lerma (México) y la mayor parte de sus tributarios son muy tóxicos (Carreño et al., 2018), así como la evaluación del riesgo toxicológico de los sedimentos en ríos (Castro-català et al., 2015) y puertos (Mccready et al., 2004) lo que puede conllevar a una gestión ambiental para mejorar las condiciones de este sistema fluvial. En 1997 este método fue utilizado para determinar que el aumento de la influencia de la luz ultravioleta causado por el agujero de la capa de ozono influye en la toxicidad de los contaminantes presentes en los sedimentos y los modifica fotolíticamente afectando la vida marina Antártica (Cleveland et al.,1997).

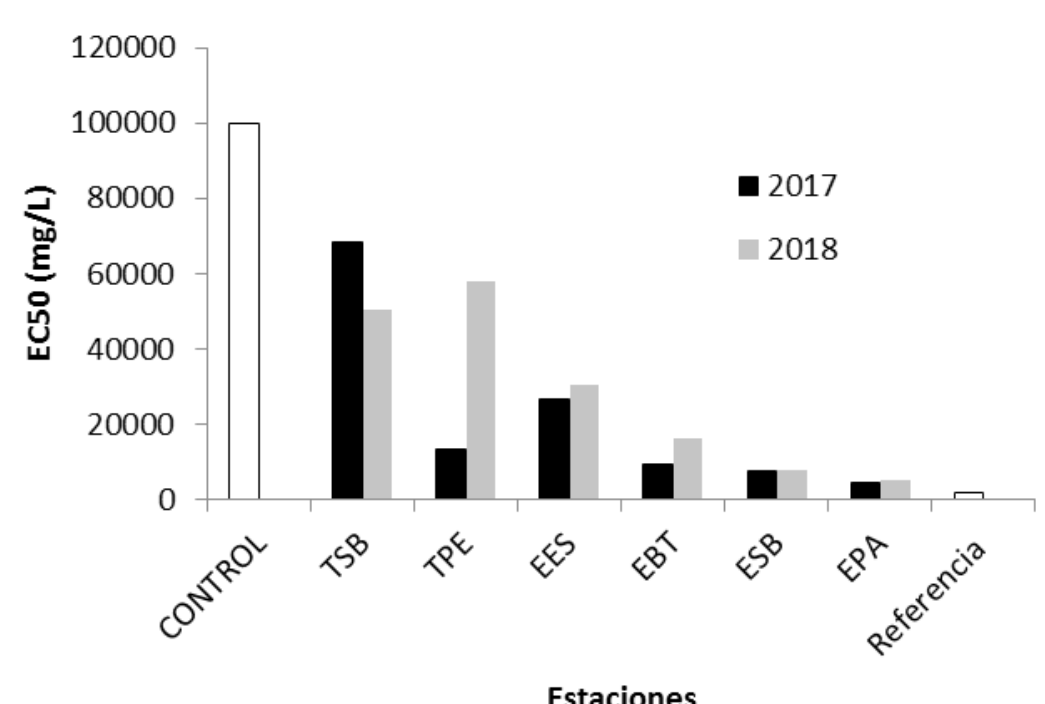

Figura 4. Toxicidad de sedimentos en las estaciones de muestreo.

Fuente: Autores

\section{CONCLUSIONES}

El sistema de medición de toxicidad con Vibrio fischeri fue eficaz para evaluar contaminantes en las matrices agua y sedimentos.

Este estudio contribuye al monitoreo de la calidad de agua del embalse $\mathrm{La} F \mathrm{Fe}$, y los resultados de estas evaluaciones sugieren que el agua y los sedimentos no presentan toxicidad, y presentan buenas condiciones, por lo que se minimizan los riesgos en la utilización del agua para su potabilización.
No se observaron variaciones significativas en las mediciones de toxicidad realizadas a las muestras de agua y sedimentos entre 2017 y 2018 a excepción de las muestras de sedimentos de la estación TPE, donde se observó un incremento de la toxicidad en el periodo 2017 - 2018.

Se evidenció potencial toxicidad en sedimentos recolectados en las estaciones de bombeo del río Pantanillo (EPA) y del tributario Palmas Espíritu Santo (TPE), por lo que se recomienda 
realizar un estudio más exhaustivo y establecer un programa de monitoreo de la calidad de agua y los sedimentos en esos sitios.

\section{CONTRIBUCIÓN DE LA AUTORÍA}

Primer autor: metodología, trabajo de campo y laboratorio, investigación, análisis de datos, conceptualización, escritura - borrador original. Segundo autor: investigación, trabajo de campo, conceptualización, revisión y edición.

\section{AGRADECIMIENTOS}

Los autores agradecemos al Departamento de Ciencia y Tecnología de Colombia, Colciencias, por el apoyo con la beca doctoral de la convocatoria 727 de 2015, así como a la Empresas Públicas de Medellín (EPM) por facilitar el acceso a sus instalaciones.

\section{LITERATURA CITADA}

Abbas, M., Adil, M., Ehtisham-ul-haque, S., Munir, B., \& Yameen, M. (2018). Vibrio fischeri bioluminescence inhibition assay for ecotoxicity assessment: A review Science of the Total Environment Vibrio fi scheri bioluminescence inhibition assay for ecotoxicity assessment : A review. Science of the Total Environment, 626, 1295-1309. https://doi.org/10.1016/j.scitotenv.2018.01.066

Allan, I. J., Harman, C., Kringstad, A., \& Bratsberg, E. (2010). Effect of sampler material on the uptake of PAHs into passive sampling devices. Chemosphere, 79(4), 470-475. https://doi. org/10.1016/j.chemosphere.2010.01.021

APHA (American Public Health A). (2017). Standars methods for the examination of water and wastewaters (23th edition).

Arias, J, C. Ramiréz, J, J. (2009). Caracterización preliminar de los sedimentos de un embalse tropical : Caracterizaci on represa La Fe (El Retiro, Antioquia, Colombia). Limnetica, 28(1), 65-78.

Beg, K. R., \& Ali, S. (2008). Microtox Toxicity Assay for the Sediment Quality Assessment of Ganga River. American Journal of Environmental Sciences, 4(4), 383-387. https://doi.org/10.3844/ ajessp.2008.383.387
Blaha, L., Hilscherova, K., Cap, T., Klanova, J., Machat, J., Zeman, J., Holoubek, I. (2009). Kinetic bacterial bioluminescence assay for contact sediment toxicity testing: Relationship with the matrix composition and contamination. Environmental Toxicology and Chemistry, 29(3), 507514. https://doi.org/10.1002/etc. 81

Burton, G, A., Baudo, R., Beltrami, M., \& Rowland, C. (2001). Assessing sediment contamination using six toxicity assays. Journal of Limnology, 60(2), 263267. https://doi.org/10.4081/jlimnol.2001.1.263

Carreño, C., Zarazúa, G., Fall, C., Ávila-Pérez, P., Tejeda, S. (2018). Evaluación de la toxicidad de los sedimentos del curos alto del río lerma, méxico. Revista Internacional de Contaminación Ambiental, 34(1), 117-126. https://doi.org/10.20937/ RICA.2018.34.01.10

Castro-català, N. De, Kuzmanovic, M., Roig, N., Sierra, J., Ginebreda, A., Barceló, D., ... Muñoz, I. (2015). Ecotoxicity of sediments in rivers : Invertebrate community, toxicity bioassays and the toxic unit approach as complementary assessment tools. Science of the Total Environment, 540, 297-306. https://doi.org/10.1016/j. scitotenv.2015.06.071

Charriau, A., Lissalde, S., Poulier, G., Mazzella, N., Buzier, R., \& Guibaud, G. (2016). Overview of the Chemcatcher $\AA$ for the passive sampling of various pollutants in aquatic environments Part $A$ : Principles, calibration, preparation and analysis of the sampler. Talanta, 148, 556-571. https:// doi.org/10.1016/j.talanta.2015.06.076

Cleveland, L. Little, E. Petty, J.D. Johnson, T. Lebo, J. A., Orazio, C. E., Jane, D. ., \& Crockett, A. (1997). Toxicological and Chemical Screening of Antarctica Sediments : Use of Whole Sediment Toxicity Tests, Microtox, Mutatox and Semipermeable Membrane Devices ( SPMDs ). Marine Pollution Bulletin, 34(3), 194-202. https://doi. org/10.1016/S0025-326X(96)00088-4

Fernández-piñas, F., Rodea-palomares, I., Leganés, F., González-pleiter, M., \& Muñoz-martín, M. A. (2014). Evaluation of the Ecotoxicity of Pollutants with Bioluminescent Microorganisms (Vol. 2). https://doi.org/10.1007/978-3-662-43619-6

Florez, M.T, Parra, L.N, Bolaños, S.V, Gallo, L.J, Poveda, A. Agudelo, D, M. (2017). Caracterización de los sedimentos de tres embalses de empresas públicas de medellín, Colombia. Politécnica, 24, 49-63. https://doi.org/10.33571/rpolitec 
Forsberg, N. D., Smith, B. W., Sower, G. J., \& Anderson, K. A. (2014). Predicting Polycyclic Aromatic Hydrocarbon Concentrations in Resident Aquatic Organisms Using Passive Samplers and Partial Least-Squares Calibration. Environ. Sci Technol., 48, 6291-6299. https://doi.org/10.1021/ es5000534

Fulladosa, E., Murat, J. C., Martınez, M., \& Villaescusa, I. (2005). Patterns of metals and arsenic poisoning in Vibrio fischer bacteria. Chemosphe$r e, 60,43-48$.

Fulladosa, E., Murat, J. C., \& Villaescusa, I. (2005). Effect of Cadmium (II), Chromium (VI), and Arsenic (V) on Long-Term Viability- and GrowthInhibition Assays Using Vibrio fischeri Marine Bacteria. Archives of Environmental Contamination and Toxicology, 306, 299-306. https://doi. org/10.1007/s00244-004-0170-5

Gatidou, G., Stasinakis, A. S., \& Iatrou, E. I. (2014). Assessing single and joint toxicity of three phenylurea herbicides using Lemna minor and Vibrio fischeri bioassays. Chemosphere, 119, 569-574.

Gonçalves, A. D., Robaina, N. F., dos Reis, L. G. T., \& Cassella, R. J. (2015). Optimization of a methodology for sampling of five polycyclic aromatic hydrocarbons in saline waters using a semipermeable membrane device. Microchemical Journal, 122, 96-101. https://doi.org/10.1016/j. microc.2015.04.008

Grigson, S., Cheong, C., \& Way, E. (2006). Studies of produced water toxicity using luminescent marine bacteria. Environmental Toxicology, 10, 111-121. https://doi.org/10.2495/ETOX060111

Grinten, E. Van Der, Pikkemaat, M. G., \& Brandhof, E. Van Den. (2010). Comparing the sensitivity of algal, cyanobacterial and bacterial bioassays to different groups of antibiotics. Chemosphere, $80(1), 1-6$.

Huckins, J. Petty, J. Booij, K. (2006). Monitors of organic chemicals in the environment Semipermeable membrane devices. New York: Springer.

Huckins, J.N. Petty, J, ID. Prest, H, F. Clark, R, C. Alvarez, D, A. Orazio, C, E. Lebo, J, A. Cranor, W, L. Johnson, B, T. (2000). A guide for the use of Semipermeable membrane devices (SPMDs) as samplers of waterborne hydrophobic organic contaminants. (C. E. R. Center, Ed.). New Haven Road, Columbia: American petroleum institute.
Jarque, S., Masner, P., Klánová, J., Prokeš, R., \& Bláha, L. (2016). Bioluminescent Vibrio fischeri Assays in the Assessment of Seasonal and Spatial Patterns in Toxicity of Contaminated River Sediments. Frontiers in Microbiology, 7(November), 1-11. https://doi.org/10.3389/fmicb.2016.01738

Ji, J., Xing, Y., Ma, Z., Zhang, M., \& Zheng, P. (2013). Acute toxicity of pharmaceutical wastewaters containing antibiotics to anaerobic digestion treatment. Chemosphere, 91(8), 1094-1098.

Johnson, B. T. (2005). Microtox acute toxicity test. In J. F. Blaise, C., Ferars (Ed.), Small-scale Freshwater Toxicity. volume 1: Toxicity test methods (pp. 69-107). Netherlands.

Johnson, B. T., Huckins, J. N., Petty, J. D., \& Clark, R. C. (2000). Collection and Detection of Lipophilic Chemical Contaminants in Water, Sediment, Soil, and Air SPMD-TOX. Environmental Toxicology, 15, 248-252. https://doi.org/10.1002/15227278(2000)15:3<248::AID-TOX10>3.0.CO;2-Q

Karacık, B., Okay, O. S., Henkelmann, B., Pfister, G., \& Schramm, K. (2013). Water concentrations of PAH , PCB and OCP by using semipermeable membrane devices and sediments. Marine Pollution Bulletin, 70, 258-265.

Koci, V., Mlejnek, M., \& Kochankova, L. (2003). Toxicological evaluation of exposed SPMD membranes. Central European Journal of Chemistry, 1(1), 28-34. https://doi.org/10.2478/BF02479255

Kovats, N., Acs, A., Goloncser, F., Barbas, A. (2011). Quantifying of bactericide properties of medicinal plants. Plant Signaling \& Behavior, 6(6), 777779.

Kováts, N., \& Horváth, E. (2016). Bioluminescencebased assays for assessing eco- and genotoxicity of airborne emissions. Luminescence, 2016, 918-923.

Kwan, K. K., \& Dutka, B. J. (1996). Development of Reference Sediment Samples for Solid Phase Toxicity Screening Tests. Bulletin of Environmental Contamination and Toxicology, 56, 696-702. https://doi.org/10.1007/s001289900102

Lohmann, R., Booij, K., Smedes, F., \& Vrana, B. (2012). Use of passive sampling devices for monitoring and compliance checking of POP concentrations in water. Environmental Science and Pollution Research, 19(6), 1885-1895. 
Mansour, S. A., Abdel-hamid, A. A., \& Ibrahim, A. W. (2015). Toxicity of Some Pesticides, Heavy Metals and Their Mixtures to Vibrio fischeri Bacteria and Daphnia magna : Comparative Study. Journal of Biology and Life Science, 6(2), 221-240.

Mccready, S., Spyrakis, G., Greely, C. R., Birch, G. F., \& Long, E. R. (2004). Toxicity of surficial sediments from sydney harbour and vicinity, australia. Environmental Monitoring and Assessment, 96, 53-83. https://doi.org/10.1023/ B:EMAS.0000031716.34645.71

Narvaez, J, F. Molina, F. (2012). Los muestreadores pasivos: Una alternativa metodologíca en el monitoreo de plaguicidas en ambientes acuaticos a nivel de sub-trazas. Revista Politecnica, 15, 53-62.

Narvaez, J. F., Lopez, V. C. A., \& Molina, F. J. (2013). Passive sampling in the study of dynamic and environmental impact of pesticides in water Muestreadores pasivos en el estudio de la dinámica de plaguicidas y el impacto ambiental en el agua. Revista Facultad de Ingenieria Universidad de Antioquia, 68, 147-159.

Onorati, F., \& Mecozzi, M. (2004). Effects of two diluents in the Microtox toxicity bioassay with marine sediments. Chemosphere, 54, 679-687.

Palma, P., Palma, V, L., Fernandes, R, M., SOares A, M., Barbosa, I, R. (2008). Acute Toxicity of Atrazine, Endosulfan Sulphate and Chlorpyrifos to Vibrio fischeri, Thamnocephalus platyurus and Daphnia magna, Relative to Their Concentrations in Surface Waters from the Alentejo Region of Portugal. Bulletin of Environmental Contamination and Toxicology, 81, 485-489.

Palma, P., Alvarenga, P., Palma, V., Matos, C., Fernandes, R. M., Soares, A., \& Barbosa, I. R. (2010). Evaluation of surface water quality using an ecotoxicological approach: A case study of the Alqueva Reservoir (Portugal). Environmental Science and Pollution Research, 17(3), 703-716.

Petty, J. D., Jones, S. B., Huckins, J. N., Cranor, W. L., Parris, J. T., McTague, T. B., \& Boyle, T. P. (2000). An approach for assessment of water quality using semipermeable membrane devices (SPMDs) and bioindicator tests. Chemosphere, 41(3), 311-321. https://doi.org/10.1016/S00456535(99)00499-3

Sabaliunas, D., Lazutka, J. R., \& Sabaliuniene, I. (2000). Acute toxicity and genotoxicity of aquatic hydrophobic pollutants sampled with semipermeable membrane devices. Environmental PoIlution, 109(2), 251-265. https://doi.org/10.1016/ S0269-7491(99)00259-6

Salazar, M. (2017). Water Distribution and Drainage Systems of Aburrá Valley, Colombia - Empresas Públicas de Medellín E.S.P. Procedia Engineering, 186, 4-11. https://doi.org/10.1016/j. proeng.2017.03.201

Svenson, A., Edsholt, E., Remberger, M., \& Rottorp, J. (1996). Sediment Contaminants and Mierotox Toxicity Tested in a Direct Contact Exposure Test, 11, 293-300. https://doi.org/https://doi.org/10.1002/ (SICI)1098-2256(1996)11:4\%3C293::AIDTOX2\%3E3.0.CO;2-4

USGS, G. S. (2014). Biological Water-Quality Assessment of Selected Streams in the Milwaukee Metropolitan Sewerage District Planning Area of Wisconsin, 2007 Investigations Report 2010 5166.

WHO. (1997). Guidelines for drinking-water quality, surveillance and control of community supplies (Vol. 3).

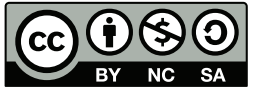


